\title{
A PROGRAM OF INFRARED ASTROMETRY
}

\author{
A.S. KHARIN \\ Main Astronomical Observatory National \\ Academy of Sciences of Ukraine \\ Goloseevo, Kiev-22, 252650 \\ Ukraine
}

There exist no special astrometric telescopes or devices suited to determine precise positions, proper motions and parallaxes in the infrared. Only astrophysical IR observations are carried out now in the range of 1-350 $\mu \mathrm{m}$. But many astronomical problems concerning galactic structure and kinematics and others may be solved if astrometric instruments and methods are developed and implemented for IR observations. Development of new technology and infrared array receivers gives the hope that many astrometric problems in the infrared would be solved successfully.

The main contents of the proposed program consist of the three tasks:

1. Construction and manufacturing of IR astrometric instruments.

2. Creation and extension of an IR reference catalogue.

3. Connection of optical, IR and radio coordinate systems.

Besides of the above mentioned problems it is necessary to point out two important scientific problems that may be solved after precise IR astrometric instruments and methods are developed.

- Finding of close double stars or multiple systems that have different coordinates in infrared and visual or in IR and radio ranges. It is obvious that these stars must be excluded from all precise reference catalogues optical, infrared and radio.

- Finding planetary systems of the nearest stars.

The proposed program must be coordinated with all other IR astronomical programs. Possibly some of these may be used also to fulfill a part of the above astrometric problems. For example the observations planned with the DENIS project could also be used for more precise position determinations of many stars in the I,J,K bands. A large (about 2 million stars) preliminary IR reference catalogue should be prepared. 